\title{
Dosis de polvo de roca y sus efectos sobre el rendimiento del tomate
}

\section{Rock dust dose and its effects on commercial performance of tomato}

\author{
Cipriano Ramon Enciso-Garay* ${ }^{1}$, Oscar Duarte Alvarez ${ }^{2}$, Giovanni Abrahám Bogado ${ }^{3}$, Victoria Rossmary Santacruz Oviedo ${ }^{4}$
}

Resumen: - La agricultura sustentable juega un rol muy importante en la producción continua de alimentos, donde se busca reducir la dependencia de fertilizantes minerales y el incremento del uso de fuentes naturales de nutrientes, con bajos impactos en el medio ambiente. Se ha evaluado el efecto de diferentes dosis de polvo de roca de origen basáltico en las características productivas del tomate cultivar Carina, a través de un experimento en el municipio de Caazapá, Departamento de Caazapá, Paraguay, en el periodo de 11/2012 a 04/2013. Los tratamientos consistieron en testigo sin fertilización, testigo experimental con fertilización química (basado en el análisis de suelo) y diferentes dosis de polvo de roca de 0,$5 ; 0,75 ; 1,0 ; 1,25 ; 1,5$ y 2,0 t ha $^{-1}$. El diseño experimental fue de bloques completos al azar con tres repeticiones. Cada unidad experimental estuvo constituida de 24 plantas, de las cuales fueron evaluadas ocho. Las variables medidas fueron masa media de frutos, rendimiento comercial y número comercial de frutos por planta (> $50 \mathrm{~g}$ ). La comparación de medias por la prueba de Tukey al $5 \%$ de probabilidad arrojó que la fertilización química resultó en mayor rendimiento, masa media de frutos y número de frutos por planta. A partir de la dosis de polvo de roca de $1,0 \mathrm{t} \mathrm{ha}^{-1}$, se encontró efecto significativo sobre el número de frutos por planta y desde $0,75 \mathrm{t} \mathrm{ha}^{-1}$ sobre la masa de frutos y rendimiento, obteniéndose, los mejores resultados con las dosis más elevadas. El análisis de regresión realizado con las dosis de polvo de roca muestra una respuesta lineal creciente para las variables evaluadas.

Palabras clave: Solanum lycopersicum L; fertilización natural; basalto; producción.

Abstract: Sustainable agriculture plays a very important role for continuous production of food, where it seeks to reduce the dependence of mineral fertilizers and the increase in the use of natural sources of nutrients, with low impact on the environment. The effect of different doses of rock dust in the productive characteristics of the Carina tomato variety was evaluated through an experiment in Caazapá, Paraguay, in the period 11/2012 to 04/2013. The treatments consisted of: a control without fertilization, experimental control with chemical fertilization (based on soil analysis) and different doses of rock dust: $0.5,0.75,1.0,1.25,1.5$ and $2.0 \mathrm{t} \mathrm{ha}^{-1}$. The experimental design was complete randomized blocks with three replications. Each experimental unit consisted of 24 plants, of which eight were evaluated. The measured variables were fruit mass, yield and commercial number of fruits per plant (>50 g); means were compared using the Tukey test at $5 \%$ probability. Chemical fertilization resulted in higher yields, increased average fruits mass and number of fruits per plant. From the $1.0 \mathrm{t}$ ha ${ }^{-1}$ rock dust dose a significant effect on the number of fruits per plant was found, as well as from the $0.75 \mathrm{tha}^{-1} \mathrm{dose}$ on the mass of fruits and yield, obtaining the best results with higher doses. There was an increasing lineal response of the evaluated variables with increasing application of the rock dust.

Key words: Solanum lycopersicum L. natural fertilization; basalt; yield.

\footnotetext{
*Autor para correspondência

Recebido para publicação em 02/02/2016; aprovado em 20/03/2016

${ }^{1}$ Prof. Dr., Universidad Nacional de Asunción, Facultad de Ciencias Agrarias, San Lorenzo, PY. E-mail: crenciso@ hotmail.com

${ }^{2}$ Prof. M.Sc., Universidad Nacional de Asunción, Facultad de Ciencias Agrarias, Caazapá, PY. E-mail: oscarduartea@gmail.com

${ }^{3}$ Ing. Agr., Universidad Nacional de Asunción, Facultad de Ciencias Agrarias, Caazapá, PY. E-mail: giovanniagro28@hotmail.com

${ }^{4} \mathrm{Dr}^{\mathrm{a}}$,, Universidad Nacional de Asunción, Facultad de Ciencias Agrarias, San Lorenzo, PY. E-mail: vrossmary@agr.una.py
} 


\section{INTRODUCCION}

Las hortalizas son altamente exigentes en nutrientes e intensivos en el uso del suelo, razón por la cual, es necesario el aporte continuo de fertilizantes para mantener el rendimiento y la calidad de la producción. Entre las más exigentes en la fertilización química se encuentra el tomate, que extrae entre 2,8 y $3,7 \mathrm{~kg}$ de nitrógeno y 3,5 a $2,5 \mathrm{~kg}$ de $\mathrm{K}_{2} \mathrm{O}$ por tonelada de fruto producido (CASTAGNINO, 2009). Alvarenga (2004), menciona que dicho cultivo, para obtener una producción de $102 \mathrm{t} \mathrm{ha}^{-1}$, extrae del suelo $150,31 \mathrm{~kg}$ de $\mathrm{N}$; $28,12 \mathrm{~kg}$ de $\mathrm{P} ; 354,06 \mathrm{~kg}$ de $\mathrm{K} ; 193,43 \mathrm{~kg}$ de Ca y 40,93 kg de $\mathrm{Mg}$. Estos datos son indicativos de la gran cantidad de nutrientes extraídos del suelo para obtener elevados rendimientos.

En el Paraguay el tomate es la hortaliza más cultivada y consumida, sin embargo la productividad es baja. Entre las posibles causas puede mencionarse que el cultivo se realiza en suelos con muchos años de uso y degradados, de baja fertilidad natural y donde también se suma los altos costos de los fertilizantes químicos solubles. En los últimos años, debido al elevado costo de los fertilizantes químicos, las investigaciones han sido orientadas a buscar nuevas alternativas, entre ellas la utilización de productos de origen natural, que además de disminuir los precios, presentan características deseables debido a que son renovables y reducen la contaminación ambiental.

Entre los fertilizantes de origen natural se encuentra el polvo de roca, que es una opción a los fertilizantes solubles (FYFE et al., 2006), que tiene la capacidad de suministrar al suelo macro y micronutrientes (KNAPIK; ANGELO, 2007), de liberación lenta (HARLEY; GILKES, 2000) y puede ser utilizado como fuente alternativa a la fertilización química. Melo et al. (2012) y Escosteguy y Klant (1998), reportaron resultados positivos a nivel de campo al utilizar dosis elevadas de polvo de roca, debido a que suministran niveles aceptables de nutrientes disponibles al suelo. De acuerdo a Atungwu et al. (2014) el polvo de roca es un fertilizante no sintético obtenido de roca finamente triturada, que contiene minerales y oligoelementos, muy utilizado en la agricultura ecológica. Trabajos de investigación desarrollados con polvo de roca por Gomes et al. (2009) en batata, Resende et al. (2006) y Souza et al. (2013) en maíz; Ferreira et al. (2009) en habilla (Phaseolus vulgaris) y Kosera et al. (2009) en poroto Adzuki; Pereira et al. (2012) en achicoria (Cichorium intibus L.); Prates et al. (2012) en la producción de mudas de Jatropha curcas L.; Sampaio et al. (2008) mudas de tomate y Camargo et al. (2012) en la producción de frutilla, indican la viabilidad de su utilización como fertilizante.

Entre los beneficios de la utilización del polvo de roca se mencionan el lento suministro de macro y micronutrientes, aumentos en la disponibilidad de los nutrientes en el suelo, reequilibrio del $\mathrm{pH}$ del suelo, aumento de la actividad microbiana y de lombrices, de la cantidad y calidad de humus, control de la erosión del suelo debido al mejor desarrollo de las plantas cultivadas y del aumento de la materia orgánica del suelo, aumento de la reserva nutricional del suelo, de la resistencia de las plantas a la acción de plagas, enfermedades, sequias y heladas, debido a la mejoría de su estado nutricional, disminución de la dependencia de fertilizantes, pesticidas y herbicidas, cuya producción exige un elevado consumo de energía (MELAMED et al., 2007) y su efecto residual prolongado (COLA; SIMAO, 2012).

Teniendo en cuenta la disponibilidad del fertilizante polvo de roca basáltico de origen nacional y la posibilidad de convertirse en una alternativa en la fertilización en hortalizas, con menor costo de producción, con bajo impacto en el ambiente $\mathrm{y}$, la falta de informaciones sobre la cantidad adecuada que debe aplicarse, se ha realizado este trabajo para evaluar el efecto de diferentes dosis en las características productivas y rendimiento del cultivo de tomate.

\section{MATERIAL Y MÉTODOS}

La investigación se desarrolló entre los meses de noviembre de 2012 y abril de 2013, en condiciones de campo en una finca del municipio de Caazapá, Departamento de Caazapá, Paraguay, situada a $26^{\circ} 11^{\prime} 33,36^{\prime}$ ' Sur y $56^{\circ}$ 22'15,4' Oeste y altitud de $143 \mathrm{msnm}$.

El clima de la región es subtropical húmedo y mesotérmico, con una precipitación media anual de $1600 \mathrm{~mm}$. La distribución mensual de lluvias es bimodal, se presenta un pico en los meses de octubre y noviembre y otro en los meses de abril y mayo. Los meses de menor precipitación corresponden a julio y agosto. La precipitación media mensual varía de $74 \mathrm{~mm}$ en el mes de julio, hasta $184 \mathrm{~mm}$ en el mes de octubre. La temperatura media anual es de $23,2{ }^{\circ} \mathrm{C}$ y la mensual oscila entre $18,5^{\circ} \mathrm{C}$ en junio y $27,05^{\circ} \mathrm{C}$ en enero, donde se presenta también las temperaturas máximas extremas.

El suelo pertenece al orden Ultisol (LÓPEZ et al., 1995) y los resultados del análisis de suelo de la parcela experimental arrojó los siguientes resultados: 1,75\% de materia orgánica, 6,35 de $\mathrm{pH} ; 2,65 \mathrm{mg} \mathrm{kg}^{-1}$ de Fósforo; 0,7 $\mathrm{cmol}_{\mathrm{c}} \mathrm{kg}^{-1}$ de Calcio; 0,6 $\mathrm{cmol}_{\mathrm{c}} \mathrm{kg}^{-1}$ de Magnesio; 0,13 $\mathrm{cmol}_{\mathrm{c}}$ $\mathrm{kg}^{-1}$ de Potasio; 0,6 $\mathrm{cmol}_{\mathrm{c}} \mathrm{kg}^{-1}$ de $\mathrm{Al}^{+3}+\mathrm{H}^{+}$y textura franco arenosa.

Los tratamientos consistieron en las diferentes fertilizaciones: testigo sin fertilización, testigo experimental con fertilización química (basado en el análisis de suelo) y diferentes dosis de polvo de roca de 0,$5 ; 0,75 ; 1,0 ; 1,25 ; 1,5$ y 2,0 $\mathrm{t} \mathrm{ha}^{-1}$. El diseño experimental fue de bloques completos al azar con tres repeticiones. Cada unidad experimental estuvo constituida por 24 plantas, de las cuales fueron evaluadas ocho, de la hilera central. El cultivar de tomate utilizado fue el hibrido Carina, perteneciente al tipo Santa Cruz, con hábito de crecimiento indeterminado.

En el tratamiento con fertilización química se aplicó de base $25 \mathrm{~g} \mathrm{planta}^{-1}$ la fórmula 10-20-10 (NPK) y en cobertura $20 \mathrm{~g}$ planta ${ }^{-1}$ cada 20 días, hasta completar $100 \mathrm{~g} \mathrm{planta}^{-1}$ el fertilizante de la fórmula 12-12-17-2 (NPKMg). En los tratamientos con polvo de roca, el $80 \%$ de la dosis fue aplicada en el momento del trasplante en los surcos y el $20 \%$ restante al inicio de la primera floración, que fue a los 19 días después del trasplante. El polvo de roca utilizado proviene de formaciones basálticas, procesado industrialmente en el distrito de Caazapá y comercializado por una empresa local. Posterior a la molienda, es pasado por un tamiz de 300 mesh (Tabla 1).

Las mudas de tomate fueron producidas en bandejas de 128 celdas y trasplantadas con cuatro hojas a una distancia de $1,0 \mathrm{~m} \times 0,5 \mathrm{~m}$, bajo cobertura de malla de color negro, con $50 \%$ de retención de luz. Se realizaron los cuidados culturales 
como tutorado, atado y colocación de cobertura orgánica muerta sobre el suelo entre las hileras y riego. Las plantas fueron conducidas con un solo tallo, eliminando todos los brotes axilares del tallo principal durante el desarrollo del cultivo.

Tabla 1- Composición química del polvo de roca utilizado en el experimento.

\begin{tabular}{|c|c|c|c|c|c|c|c|c|c|c|c|c|}
\hline $\bar{P}$ & $\bar{K}$ & $\mathrm{Ca}^{+2}$ & $\mathrm{Mg}$ & $\mathrm{AI}^{*}$ & $\mathrm{Fe}$ & $\bar{B}$ & $\mathrm{Mn}$ & $\mathrm{Zn}$ & $\mathrm{Cu}$ & $\mathrm{Ni}$ & Mo & $\mathrm{Si}$ \\
\hline \multicolumn{13}{|c|}{$\mathrm{cmol}_{\mathrm{c}} / \mathrm{kg}$} \\
\hline 3,52 & 6,13 & 45,16 & 12,89 & 0,006 & 23,41 & 0,67 & 0,46 & 0,181 & 0,074 & 0,01 & 0,0015 & 54,02 \\
\hline
\end{tabular}

La cosecha se efectuó a medida que los frutos alcanzaron la madurez fisiológica, en forma escalonada, en 10 oportunidades durante 55 días.

Se evaluó la masa media de frutos, rendimiento comercial y número comercial de frutos por planta (>50 g). Los datos colectados fueron sometidos a análisis de varianza y a la comparación de medias por la prueba de Tukey al 5\% de probabilidad. Los efectos de las dosis de polvo de roca fueron evaluadas por medio del análisis de regresión, adoptándose como criterio para la selección del modelo, el efecto significativo de la prueba de $\mathrm{F}$ al $5 \%$ de probabilidad.

\section{RESULTADOS Y DISCUSION}

La composición química del polvo de roca (Tabla 1), muestra un alto contenido de macro y micronutrientes que influenciaron la producción del tomate. Se obtuvieron diferencias estadísticas para todas las variables evaluadas, entre el testigo absoluto, el testigo con fertilización química y los niveles de polvo de roca.

Analizando el número de frutos por planta obtenido en función a los diferentes tratamientos (Tabla 2), se observó que los tratados con 1,5 y $2,0 \mathrm{t} \mathrm{ha}^{-1}$ de polvo de roca fueron iguales entre sí estadísticamente, sin diferir del tratamiento con fertilización química; sin embargo presentaron mayor número de frutos, cuando comparados con los demás tratamientos que recibieron polvo de roca y el testigo absoluto. Los tratados con dosis de polvo de roca de 1,0 y $1,25 \mathrm{t} \mathrm{ha}^{-1}$ promovieron valores intermedios y difirieron del testigo sin fertilización y de los tratados con 0,5 y $0,75 \mathrm{t} \mathrm{ha}^{-1}$, en los que se obtuvieron menor número de frutos por planta. En trabajos realizados por Atungwu et al. (2014) la aplicación de polvo de roca en dosis de $2,5 \mathrm{t} \mathrm{ha}^{-1}$, en el cultivo de sandía, promovió el aumento de la floración y controló la aparición de nematodos. Esta investigación se desarrolló en el periodo de temperaturas elevadas por lo que puede decirse que la utilización del fertilizante natural favoreció la floración y desarrollo posterior, siendo menos afectado por problemas de aborto, común en esta época.

Para la masa de frutos, se obtuvo la mayor media en el tratamiento con fertilización química $(142,75 \mathrm{~g})$, difiriendo de los demás. El tratamiento con la dosis de polvo de roca de 2,0 $\mathrm{t}$ ha $^{-1}$ ocupó el segundo lugar, pero sin diferir estadísticamente de los tratados con 1,25 y 1,5 $\mathrm{t} \mathrm{ha}^{-1}$. La dosis menor de polvo de roca, no difirió del testigo absoluto y ambos tratamientos presentaron las menores medias, indicando que dosis bajas de polvo de roca no contribuyen para el aumento de la masa de frutos.

Tabla 2. Número de frutos, masa de frutos y rendimiento, por planta, del tomate con fertilización química y diferentes dosis de polvo de roca. Caazapá, PY. 2013.

\begin{tabular}{lccc}
\hline Tratamientos & $\begin{array}{l}\text { Número de frutos } \\
\text { planta }^{-1}\end{array}$ & $\begin{array}{c}\text { Masa de frutos } \\
\left(\mathrm{gramos} \mathrm{planta}^{-1}\right)\end{array}$ & $\begin{array}{c}\text { Rendimiento } \\
\left(\mathrm{kg} \mathrm{planta}^{-1}\right)\end{array}$ \\
\hline Testigo sin fertilización & $34 \mathrm{c}^{*}$ & $96,22 \mathrm{~d}$ & $3,24 \mathrm{e}$ \\
Fertilización química & $42 \mathrm{a}$ & $142,75 \mathrm{a}$ & $5,96 \mathrm{a}$ \\
& & & - \\
Dosis de polvo de roca (t ha-1) & - & - & $3,35 \mathrm{e}$ \\
0,50 & $34 \mathrm{c}$ & $99,93 \mathrm{~d}$ & $3,94 \mathrm{~d}$ \\
0,75 & $34 \mathrm{c}$ & $117,50 \mathrm{c}$ & $4,20 \mathrm{~cd}$ \\
1,00 & $36 \mathrm{~b}$ & $117,68 \mathrm{c}$ & $4,50 \mathrm{c}$ \\
1,25 & $37 \mathrm{~b}$ & $122,05 \mathrm{bc}$ & $5,25 \mathrm{~b}$ \\
1,50 & $41 \mathrm{a}$ & $128,25 \mathrm{~b}$ & $5,37 \mathrm{~b}$ \\
2,00 & $41 \mathrm{a}$ & $129,82 \mathrm{~b}$ & 2,75 \\
\hline Coeficiente de Variación $(\%)$ & 1,36 & 2,34 & \\
\hline
\end{tabular}

*Medias seguidas de la misma letra en la columna no difieren entre sí por la prueba de Tukey al 5\% de probabilidad de error.

En relación al rendimiento comercial de frutos, la mayor media presentó el tratamiento con fertilización química, con un promedio de 5,96 kg planta- ${ }^{1}$, quedando en segundo lugar los tratados con 1,75 y $2,0 \mathrm{t} \mathrm{ha}^{-1}$ que son iguales estadísticamente entre sí, con medias de 5,25 y $5,37 \mathrm{~kg}$ planta ${ }^{1}$, pero superiores al testigo absoluto y a los tratados con dosis de polvo de roca de 0,$5 ; 0,75 ; 1,0$ y $1,25 \mathrm{t} \mathrm{ha}^{-1}$. Comparando la dosis de 2 tha $^{-1}$ con el testigo sin fertilización, se obtuvo un incremento de $65 \%$ en el rendimiento comercial de frutos. El hecho de que la fertilización química sea estadísticamente superior a la dosis más elevada de polvo de roca, coincide con Zuba et al. (2011) quienes también encontraron que la fertilización química promovió mayor rendimiento en el tomate, comparado con otras formulaciones donde fue incluido el polvo de roca. La solubilidad lenta de los nutrientes del polvo de roca podría ser el motivo por el cual la fertilización química sea más eficiente en la obtención de mejores rendimientos. Según Luchese et al. (2002) los fertilizantes químicos liberan más rápidamente los nutrientes, y de esta manera atienden de forma más eficaz las exigencias 
de la mayoría de las plantas cultivadas de ciclo corto, sin embargo son más propensos a la lixiviación, contaminando el medio ambiente. De esa forma a través del agua de lluvia o del riego pueden contaminar el agua de los ríos y napas freáticas en niveles indeseables para el consumo humano, como ocurre con el nitrógeno, lixiviado en la forma de nitrato.

Para facilitar la liberación de nutrientes del polvo de roca Garcia Júnior (1991), sugiere la solubilización biológica, mediante la utilización de bacterias específicas (LOMBARDI et al., 1981). Así también, Souza et al. (2013) resaltan que la utilización de vermicompuestos con polvo de roca mejora la disponibilidad de nutrientes, en especial de micronutrientes. En otro trabajo Lopes et al. (2014) encontraron que el uso de polvo de roca mezclado con vinaza mejora la fertilidad del suelo. De acuerdo a los resultados de esta investigación a partir de la dosis de 1,0 t ha ${ }^{-1}$, se observa efecto significativo sobre el número de frutos por planta y desde $0,75 \mathrm{t} \mathrm{ha}^{-1}$ sobre la masa de frutos y rendimiento, obteniéndose, los mejores resultados para todas las variables con la dosis más elevada.

En investigación realizada por Ferreira et al. (2009) aplicando diferentes dosis de polvo de roca en el cultivo de habilla no encontraron diferencias significativas en el rendimiento en función a las dosis aplicadas, sin embargo, reportaron que la combinación de polvo de roca con estiércol bovino promovió incremento en el rendimiento. Las diferencias en los resultados con las investigaciones citadas anteriormente pueden ser atribuidas a que éste trabajo se realizó en un suelo con $1,75 \%$ de materia orgánica y $\mathrm{pH}$ de 6,35; mientras que los otros autores realizaron sus investigaciones en suelos más degradados. Por otro lado, Souza et al. (2013), encontraron que al combinar humus de lombriz con polvo de roca se incrementa la solubilización de minerales, permitiendo un mejor desarrollo del cultivo de maíz. Así mismo, en trabajos realizados con diferentes dosis de polvo de roca de origen basáltico y combinaciones con otros compuestos orgánicos como lodo, restos vegetales o estiércol, mejoraron químicamente la calidad del suelo desde el punto de vista microbiológico, favoreciendo el desenvolvimiento de la especie forestal Astronium fraxinifolium Schott (SILVA et al., 2008). Camargo et al. (2012), al combinar $0 ; 50$ y $100 \mathrm{t} \mathrm{ha}^{-1}$ de estiércol bovino con $0 ; 2 ; 4$ y $6 \mathrm{t} \mathrm{ha}^{-1}$ de polvo de roca de origen basáltico observaron que elevadas dosis de estiércol asociado con dosis intermedias de polvo de roca incrementan la productividad de la frutilla, en relación a la utilización del polvo de roca en forma aislada.

El análisis de regresión aplicado para verificar los efectos de las dosis de polvo de roca con relación a las variables de producción del cultivo de tomate estudiadas (Figura 1) muestran que la relación entre las dosis de polvo de roca y el número comercial de frutos por planta, masa de frutos y rendimiento comercial de frutos por planta es lineal y positivo, es decir aumentando las dosis de polvo de roca, también se incrementa las medias de dichas variables.
Figura 1. Número de frutos por planta, masa media de frutos y rendimiento comercial de frutos por planta del tomate variedad Carina en función a las dosis de polvo de roca. Caazapá, PY. 2013.

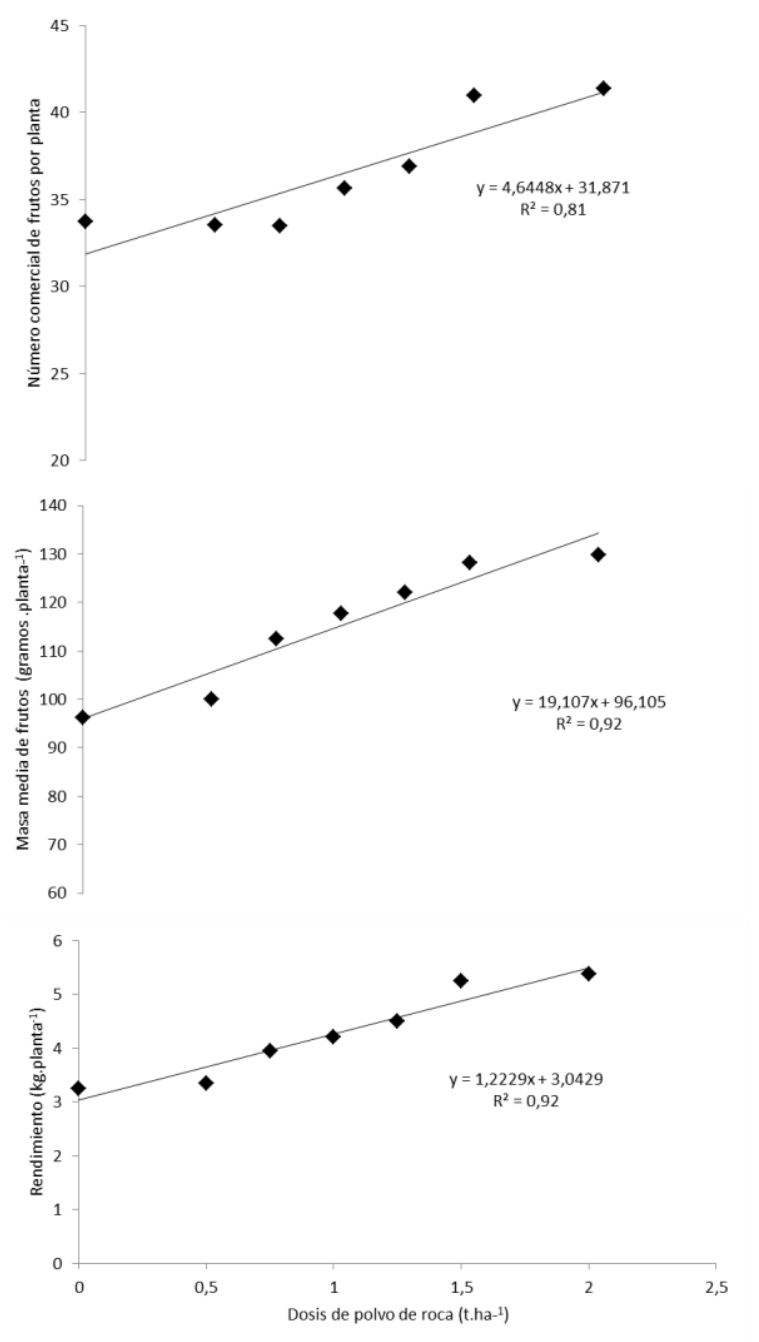

Para el número de frutos por planta por cada kilogramo de polvo de roca aplicado, aumenta el número de frutos por planta en 0,0046 unidades, mientras que la masa de frutos en $0,0191 \mathrm{~g}^{-1}$ y el rendimiento en $0,0012 \mathrm{~kg}$ planta $^{-1}$. En investigaciones realizadas por Santos et al. (2014) aplicando polvo de roca en dosis de $0 ; 1 ; 2 ; 3$ y $4 \mathrm{t} \mathrm{ha}^{-1}$ en el cultivo de papa, encontraron respuesta cuadrática para el rendimiento comercial de tubérculos, obteniendo el máximo valor con la dosis de 2,51 $\mathrm{t} \mathrm{ha}^{-1}$. Además observaron efectos positivos sobre otros componentes de producción de dicho cultivo.

De acuerdo a Melamed et al. (2007), residuos inorgánicos como el polvo de roca tienen el potencial de aumentar las reservas del suelo y favorecer la resistencia de la planta a factores de estrés biótico y abiótico por el mejoramiento de la nutrición de la planta y de esa forma aumentan la producción. Los parámetros de altura en mudas, el diámetro, la biomasa, el contenido de clorofila y la tasa fotosintética del tomate aumentaron considerablemente en experimentos realizados con aplicación de polvo de roca mezclado con fertilizante orgánico (LI; DONG, 2013). Los resultados positivos encontrados en esta investigación con la aplicación de polvo de roca pueden ser debidos a su elevado contenido en macro y micronutrientes y a la concentración de materia orgánica del suelo donde se desarrolló el 
experimento. Camargo et al. (2012) mencionan que el polvo de roca además de suministrar cantidades apreciables de nutrientes al suelo, adiciona coloides negativos, debido a la presencia de silicio. Esos coloides permiten la adsorción de cationes como $\mathrm{Ca}^{+2}, \mathrm{Mg}^{+2}$ y K, impidiendo que los mismos sean llevados por el agua. Además, el polvo de roca contiene elevado contenido de silicio que confiere a las plantas mayor resistencia al ataque de plagas y enfermedades (EPSTEIN, 2001; GOMES et al., 2009).

\section{CONCLUSIONES}

La aplicación de polvo de roca promueve aumentos significativos a partir de $1,0 \mathrm{t} \mathrm{ha}^{-1}$ en el número de frutos por planta y de $0,75 \mathrm{t} \mathrm{ha}^{-1}$ en la masa de frutos y el rendimiento del tomate.

El número de frutos por planta, la masa de frutos y la productividad presenta respuesta lineal creciente a las dosis aplicadas de polvo de roca.

El polvo de roca puede ser utilizado como alternativa a la fertilización química y el mejoramiento de la técnica de utilización y las eventuales mezclas con abonos orgánicos deben ser considerados en otros estudios.

\section{REFERENCIAS BIBLIOGRÁFICAS}

ALVARENGA, M. A. R. Tomate: produção em campo, em casa de vegetação e em hidroponía. Lavras: Universidad Federal de Lavras, 2004. 400p.

ATUNGWU, J. J.; OLADEJI, O. A.; AIYELAAGBE, I.; OLABIYI, T. I. Influence of rock dust-based soil remineralization on root-knot disease of organic watermelon plants. Archives of Phytopathology and Plant Protection, London, v.47, n.12, p.1489-1496, 2014.

CAMARGO, C. K.; DE RESENDE, J. T. V.; CAMARGO, L. K. P.; FIGUEIREDO, A. S. T.; ZANIN, D. S. Produtividade do morangueiro em função da adubação orgânica e com pó de basalto no plantio. Semina: Ciências Agrárias, Londrina, v.33, n.6, p. 2985-2994, 2012.

CASTAGNinO, A. M. Manual de cultivos hortícolas innovadores. Hemisferio Sur. Buenos Aires. 2009. 356p.

COLA, G. P. A.; SIMÃO, J. B. P. Rochagem como forma alternativa de suplementação de potássio na agricultura agroecológica. Revista Verde, Mossoró, v.7, n.4, p. 15-27, 2012.

EPSTEIN, E. Silicon in plants: facts vs concepts. In: DATNOFF, L. E.; SNYDER, G. H.; KORNDÖRFER, G. H. (eds.). Silicon in agriculture. Netherlands: Elsevier Science, Amsterdam. 2001. p.1-16.

ESCOSTEGUY, P. A. V.; KLAMT, E. Basalto moído como fonte de nutriente. Revista Brasileira de Ciência do Solo, Mossoró, v.22, n.1, p.11-20, 1998.

FERREIRA, E. R. M.; ALMEIDA, J. A.; MAFRA, A. L. Pó de basalto, desenvolvimento e nutrição do feijão comum (Phaseolus vulgaris) e propriedades químicas de um Cambissolo Húmico. Ciências Agroveterinárias, Lages, v.8, n.2, p.111-121, 2009.
FYFE, W. S.; LEONARDOS, O. H.; THEODORO, S. H. Sustainable farming with native rocks: the transition without revolution. Anais da Academia Brasileira de Ciências, Rio de Janeiro, v.78, n.4, p.715-720, 2006.

GARCIA JÚNIOR, O. Isolation and characterization of Thiobacillus thiooxidans and Thiobacillus ferrooxidans from mineral mines. Revista Brasileira de Microbiologia, São Paulo, v. 22, n. 1, p.1-6, 1991.

GOMES, F. B.; MORAES, J. C.; NERI, D. K. Adubação com silício como fator de resistência a insetos-praga e promotor de produtividade em cultura de batata inglesa em sistema orgânico. Ciência e Agrotecnologia, Lavras, v.33, n.1, p.1823. 2009.

HARLEY, A. D.; GILKES, R. J. Factors influencing the release of plant nutrient elements from silicate rock powders: a geochemical overview. Nutrient Cycling in Agroecosystems, Netherlands, v. 56, n. 1, p.11-36, 2000.

KOSERA, C.; SPADOTTO, D.; SCHLUCUBIER, L.; BORTOLINI, P. C.; MARQUEZ, A. C,; OLIVEIRA, C. D:, Produção de feijão adzuki sob diferentes doses de pó de basalto. Revista Brasileira de Agroecologia, Bananeiras. v.4, n.2, p. 4227-4231, 2009.

KNAPIK, J. G.; ANGELO, A. C. Crescimento de mudas de Prunus sellowii Koehne em resposta a adubações com NPK e pó de basalto. Floresta, Curitiba, v.37, n.2, p. 257-264, 2007.

LI, J. G.; DONG, Y. H. Effect of a rock dust amendment on disease severity of tomato bacterial wilt. International Journal of General and Molecular Microbiology, Netherlands, v.103, n.1, p.11-22, 2013.

LÓPEZ, O.; GONZÁlEZ, E.; LLAMAS, P.A. de; MOLINAS, A.S.; FRANCO, E.S.; GARCÍA, S.; RIOS, E.O. Estudio de reconocimiento de suelos, capacidad de uso de la tierra y propuesta de ordenamiento territorial preliminar de la región oriental del Paraguay. Asunción. PY. MAG. 1995. $246 \mathrm{p}$.

LOPES, O. M. M.; CARRILHO, E. N. V. M.; LOPESASSAD, M. L. R. C. Effect of rock powder and vinasse on two types of soils. Revista Brasileira de Ciência do Solo, Viçosa, v.38, n.5, 1547-1557, 2014.

LOMBARDI, M. L. C. O.; LOPES, E. S.; CARDOSO, E. J. B. N.; SILVA, M. T. R. Eficiência da dissolução de três fosfatos naturais no solo, pela atividade microbiológica de oxidação de enxofre elementar. In: Congresso brasileiro de ciência do solo, 18, Salvador. Resumos. Campinas, Sociedade Brasileira de Ciência do Solo, p. 38. 1981.

LUCHESE, E. B.; FAVERO, L. O. B.; LENZI, E. Fundamentos da química do solo: teoria e prática. 2.ed. Rio de Janeiro: Freitas Bastos, 2002. 182p.

MELAMED, R.; GASPAR, J. C.; MIEKELEY, N. Pó-derocha como fertilizante alternativo para sistemas de produção sustentáveis em solos tropicais. Brasília: CETEM/MCT, Série Estudos e Documentos, 2007. 23p. 
MELO, V. F.; UCHÔA, S. C. P.; OLIVEIRA, F. de; BARBOS, G. F. Doses de basalto moído nas propriedades químicas de um Latossolo Amarelo distrófico da savana de Roraima. Acta Amazônica, Manaus, v.42, n.4, p.471-476, 2012.

PEREIRA, D. C.; GRUTZMACHER, P.; BERNARDI, F. H.; MALLMANN, L. S.; COSTA, L. A. D. M.; COSTA, M. S. S. M. Produção de mudas de almeirão e cultivo no campo, em sistema agroecológico. Revista Brasileira de Engenharia Agrícola e Ambiental, Campina Grande, v.16, n.10, p.11001106, 2012.

PRATES, F. B. S. de; LUCAS, C. D. S. G.; SAMPAIO, R. A.; JÚNIOR, D. D. S. B.; FERNANDES, L. A.; JUNIO, G.

R. Z. Crescimento de mudas de pinhão-manso em resposta a adubação com superfosfato simples e pó-de-rocha. Revista Ciência Agronômica, Fortaleza, v.43, n.2, p.207-213, 2012.

RESENDE, A. V. de; MACHADO, C. T.; MARTINS, E. de S.; SENA, M. C. de; NASCIMENTO, M. T. do; SILVA, L. de C. R.; LINHARES, N. W. Rochas como fontes de potássio e outros nutrientes para culturas anuais. Revista Espaço \& Geografia, Brasília, v.9, n.1, p. 135-161, 2006.

SAMPAIO, R. A.; RAMOS, S. J.; GUILHERME, D. O.; COSTA, C. A.; FERNANDES, L. A. L. Produção de mudas de tomateiro em substratos contendo fibra de coco e pó de rocha. Horticultura Brasileira, Brasília, v.26, n.4, p.499-503, 2008.

SANTOS, J. F. dos; SILVA, E. D. da; BESERRA, A. C. Produção agroecológica de batata em relação à doses de pó de rocha. Tecnologia \& Ciência Agropecuária, João Pessoa, v.8, n.1, p.29-35, 2014.

SILVA, E. A. da; CASSIOLATO, A. M. R.; MALTONI, K. L.; SCABORA, M. H. Efeitos da rochagem e de resíduos orgânicos sobre aspectos químicos e microbiológicos de um subsolo exposto e sobre o crescimento de Astronium fraxinifolium Schott. Revista Árvore, Viçosa, v.32, n.2, p. 323-333, 2008.

SOUZA, M. E. P. de; CARVALHO, A. M. X. de; CÁSSIA DELIBERALI，D. de; JUCKSCH， I.; BROWN， G. G.; MENDONÇA, E. S.; CARDOSO, I. M. Vermicomposting with rock powder increases plant growth. Applied Soil Ecology, Netherlands, v.69, p.56-60. 2013.

ZUBA, S. N.; NOGUEIRA, W. C. L.; FERNANDES, L. A.; SAMPAIO, R. A.; COSTA, C. A. Yield and nutrition of tomato using different nutrient sources. Horticultura Brasileira, Brasília, v.29, n.1, p.50-56, 2011. 